\title{
STIAS-Wallenberg Roundtable on mHealth: \\ Towards a roadmap for image-based mobile technologies for health care
}

AUTHOR:

Michelle Galloway

\section{AFFILIATION:}

${ }^{1}$ Media Officer, Stellenbosch Institute for Advanced Study, Stellenbosch, South Africa

\section{CORRESPONDENCE TO: Michelle Galloway}

\section{EMAIL:}

michelle.rotchford.galloway@ gmail.com

\section{KEYWORDS:}

mobile health care;

patient-centred;

artificial intelligence;

resource-constrained

\section{HOW TO CITE:}

Galloway M. STIAS-Wallenberg Roundtable on mHealth: Towards a roadmap for image-based mobile technologies for health care. S Afr J Sci. 2017;113(7/8), Art. \#a0218, 2 pages. http:// dx.doi.org/10.17159/sajs.2017/ a0218

(C) 2017. The Author(s). Published under a Creative Commons Attribution Licence.
The fifth STIAS-Wallenberg Roundtable was held in February 2017 at the Stellenbosch Institute for Advanced Study (STIAS). The theme was 'Image-based Mobile Technology for Diagnostics and Treatment to Improve Access and Equity in Health Care'. The overarching aim was to provide a forum for stakeholders to discuss the possibilities that current developments in image-based mobile health (mHealth) offer for timely, accurate and equitable health-care delivery, as well the challenges that their development and implementation may entail for potential users and beneficiaries. The emphasis was on the need to find solutions and to map out a future roadmap for the implementation, expansion and up-scaling of image-based mHealth to improve health care, particularly in resource-constrained settings.

For the past 5 years, the Marianne and Marcus Wallenberg Foundation has supported an annual Roundtable during which representatives from South Africa, Sweden and the broader international community can engage in dialogue and debate around a central theme, typically one related to current global challenges but particularly focusing on their local manifestation. Previous Roundtable themes were 'Energy', 'Mental Health', 'Agricultural Transformation' and 'Innovation for Prosperity'.

This year's event attracted over 60 participants from 16 countries and provided an opportunity for intense crosssectoral and cross-disciplinary discussions and networking. There was representation from the clinical, research, policy, business, media and social sectors. Sessions ranged from overviews of the landscape; unpacking the practical experiences and case studies of using mHealth in clinical settings; and overcoming challenges in implementation; to barriers and facilitators; and the need for leadership and change agents. The aim was to facilitate cross-pollination rather than focus on obstacles and barriers, and to inform the wider community, in particular policymakers, about the possibilities in mHealth.

Technology in health care has obvious benefits, like improving patient management options and outcomes, as well as secondary potential benefits like fewer referrals, reduced costs and time saved. It can also assist in diminishing professional isolation and promoting recruitment and retention in rural areas. This, in turn, can contribute to more equitable systems in global health care in resource-poor settings in high-income as well as low- and middleincome countries.

However, with many mHealth projects the challenge seems to be facilitating the initial adoption and sustained implementation in the health system, a lack of consideration for the perspective of the users, and that the technologies are not used to their full potential.

The emphasis therefore throughout the Roundtable was on the need for a patient-centred or end-user focus; the need to move away from endless pilot studies to scale-up of successful interventions; the need for appropriate policy and regulation including data safety, ethical issues and the protection of patients; and, the need to ensure equity and that issues of cost and access (particularly connectivity) do not further divide the world.

The complexity of the health-care ecosystem was emphasised by a number of speakers, as was the need for enabling policies and for mHealth not to remain isolated within a specific sector. Mohammed Dalwai, Co-founder and COO of Essential EMGuidance and President of the Board of Doctors Without Borders (South Africa) focused on the need to move away from pilot studies and to start studying the clinical impact and the practicalities of scaling up successful interventions. He pointed out that often projects are not scaled up because of regulatory issues. He stressed the need for a government mHealth body and a national eHealth strategy in South Africa.

\section{Lessons from case studies}

Among the case studies presented was an interesting presentation on the use of mobile technology in the recent Ebola crisis. Ousmane Ly, Director General for eHealth in the Mali Ministry of Health, pointed out that ICT had played a substantial role in the emergency response but he also noted that many of the tools developed during the outbreak are not used anymore.

A detailed presentation was also given on an image-based app used to assist in the diagnosis of burn injuries, which brings together a multidisciplinary team from the Karolinska Institute in Sweden, the Western Cape government and the Universities of Cape Town and Stellenbosch.

The project uses a smartphone-based app to transmit images and other data of burn injuries for diagnostic, treatment and referral advice. In its most recent version, the burns component has been added to an existing app (the Vula app) which is used for other disease conditions. The app is being tested in sites in the Western Cape in South Africa (to be expanded in 2017 to encompass the whole province, as well as sites in Durban, a number of sites in Tanzania and, eventually, Ethiopia). Besides using images, the app is front-loaded with drop-down questions which aid in diagnosis and treatment decisions. This information triggers an inbuilt management protocol consisting of general care advice, and a link to an expert to provide case-specific advice.

South Africa, in particular, suffers from a quadruple burden of disease with injuries, interpersonal violence and traffic-related deaths ranking alongside HIV/AIDS and tuberculosis, infectious diseases and a rising epidemic of 
non-communicable disease. The problem of burns injuries in South Africa is big and rising. Burns are associated with shack fires as well as scalding during household chores, with the winter months regarded as 'burn season' by emergency services.

The project had to overcome some initial challenges related to comparing images from different phones and also ensuring that the quality of images from darker skins are as good as those from lighter skins. Nonetheless, current results show that the app is easy to use, does not require extensive training, and that the resulting diagnoses are as good as those based on bedside diagnosis.

Studies on the acceptability of the app as well as comparing treatment outcomes with and without the app are being undertaken. The possibility of machine-learning burn diagnostics and the creation of algorithms to offer an automated diagnosis and treatment response are also currently being investigated although this implementation remains a long way off.

\section{Users involvement in development}

A recurring theme was the need to ensure early involvement of both health-care personnel and, where possible, patients in app development with the aim of overcoming practical, on-the-ground challenges and ensuring that technology is guided by needs. Presentations by nurses Halima Adam and John Bosco Kamugisha from Uganda highlighted some of these challenges - including cost, Internet accessibility and airtime; power shortages; lack of training; use of personal phones and safety issues around phones; data accuracy and security.

\section{mHealth as the new normal}

The need for mHealth to become the new normal was emphasised by various presenters. Speaking of the future possibilities, Jan Gulliksen of KTH Royal Institute of Technology in Sweden emphasised the need for societal digitisation - not just in developing technologies for context but rather for changing the context, and increasing skills for a digital society.

Johan Lundin of the University of Helsinki added to this theme by pointing out that up to $80 \%$ of communication in future will be image based and that adding in artificial intelligence will change the face of mobile diagnostics.
The need to focus on apps that can go viral was emphasised by Stefan Fölster, Head: Reform Institute, Stockholm. He outlined four main criteria that make this possible: there should be no extra cost for the end user (so not all apps should rely on smartphones); the adopting agency should be able to recoup the costs; mHealth should not raise costs by raising demand more than the cost savings allow; and, there should not be duplication of other mHealth investments. He also pointed out the need to look for technology leaps, for example, artificial intelligence and systems that learn along the way.

Isaac Bogosh of the University of Toronto cautioned, however, about the need for quality. He pointed out that the goal of mobile microscopy must be to deliver equitable health care by bringing quality laboratory diagnosis to low-resource settings. He encouraged more validation research in real-world settings but also emphasised the need to resist the urge to build the cheapest microscopes that sacrifice image quality. He called for robust devices with sufficient optics designed for efficient throughput.

\section{Generating the roadmap}

The Roundtable group work and plenary discussions focused on five key questions:

1. How should the most important barriers to the implementation of image-based mHealth in the clinical setting be overcome?

2. How can frontline health-care workers be enabled to adopt imagebased $\mathrm{mHealth}$ in their practice?

3. Which are the key strategies to overcome organisational challenges to the implementation of image-based mHealth within the health sector?

4. Which are the key strategies to overcome technical challenges in implementing image-based mHealth within the health sector?

5. For each category of stakeholders represented, what are the most important priorities to build and sustain leadership in mHealth?

These questions generated statements which were prioritised by voting in the final session. The results will form the basis of the Roadmap which will be published and disseminated in the coming months. 